\title{
Morphological and Structural Elucidation of Room Temperature Catalytic Degradation of Amoxicillin Antibiotic Using Zinc Porphyrin Metal Organic Framework
}

\author{
Shatakshi Saxena ${ }^{1}$, Punya Saluja ${ }^{1}$, JBM Krishna ${ }^{2}$, Tinku Basu ${ }^{3, *}$ \\ ${ }^{1}$ Centre for Converging Technologies, University of Rajasthan, Jaipur, India \\ ${ }^{2}$ University Grants Commission, Department of Atomic Energy, Consortium For Scientific Research, Bidhan Nagar, Kolkata, India \\ ${ }^{3}$ Amity Centre for Nanomedicine, Amity University, Uttar Pradesh, India \\ Email address: \\ tbasu@amity.edu (T. Basu), basu002@gmail.com (T. Basu) \\ ${ }^{*}$ Corresponding author
}

\section{To cite this article:}

Shatakshi Saxena, Punya Saluja, JBM Krishna, Tinku Basu. Morphological and Structural Elucidation of Room Temperature Catalytic Degradation of Amoxicillin Antibiotic Using Zinc Porphyrin Metal Organic Framework. Advances in Materials.

Vol. 10, No. 2, 2021, pp. 23-30. doi: 10.11648/j.am.20211002.12

Received: December 7, 2020; Accepted: December 15, 2020; Published: July 13, 2021

\begin{abstract}
Environment is witnessing an extensive increase in persistent and bioactive micro pollutants from the last few decades. Since most clinical modalities are flooded with antibiotics, environmental samples such as sewage treatment effluents, surface water and secondary water drainage etc. reveal the presence of bio-recalcitrant antibiotics worldwide, ergo there is a spurring rise in antibiotic resistant microorganisms. In order to circumvent the escalating concentration of amoxicillin (microgram/liter) we contrived a potential metal organic framework (MOF) and lab tested it for catalytic functionality. A zincporphyrin MOF using Tetra (4-carboxyphenyl) porphyrin H2TCPP (>97\%) as a ligand and Zinc acetate as a metal salt was solvothermally synthesized. The morphological and structural characterization were performed using FESEM, XRD, EDAX, FTIR and UV-Visible spectroscopy. The fabricated MOF catalyst exhibits sterling ability of amoxicillin degradation in a dark environment at room temperature, accounting a degradation efficiency of about $97.3 \%$ in a $0.1 \mathrm{mg} / \mathrm{ml}$ concentration. 90 minutes of catalyst exposure to the antibiotic shows maximum degradation after which there is no further change in amoxicillin concentration. First time we have exploited electrochemical cyclic voltametric (CV) measurement to monitor the degradation process which was validated by FTIR recording, and noticed that the degradation process followed a first order kinetics. We have also discussed the morphological stability of the fabricated $\mathrm{Zn}$ porphyrin MOF after antibiotic exposure and reported a comparative outline between catalytic efficiency of different MOF species. High porosity and effective charge transfer between carboxyphenyl) porphyrin ligand to vacant $\mathrm{d}$ orbital of $\mathrm{Zn}^{+2}$ are pivotal factors for effective sorption and degradation of amoxicillin antibiotics dark at room temperature. We would expect the developed $\mathrm{Zn}$ porphyrin MOF is very promising for successful commercial application due to its energy efficiency (dark) and simple process for effective degradation of antibiotics.
\end{abstract}

Keywords: Zn Porphyrin MOF, Amoxycillin, Room Temperature Degradation, FTIR Sensing

\section{Introduction}

Micro-pollutants are toxic even in minute quantities. With the increased usage of pharmaceuticals in human and veterinary sectors, there is a rise of such contaminants in water bodies, their components remain active even after their disposal from the body which accumulate in form of effluents. These can penetrate the earth contaminating the water beds, or can transform into a new composition after losing their chemical integrity. Antibiotics are emerging as major class of bio recalcitrant pollutants in wastewater effluents [1, 2]. These even at low concentrations can develop antibiotic resistance among bacteria. This will not only affect the effectiveness of the antibiotics in use but might also result in new pathogenic strains which are more harmful $[3,4]$.

Metal Organic Frameworks (MOF) are 3-D network forming materials supporting an ultra-porous structure, that 
belongs to a class of crystalline micro-mesoporous hybrid materials. These have drawn extensive attention in the past years because of the enormous surface area, porosity, structural tunebility and their ability to behave as heterogeneous photo catalysts that can be recycled $[5,6]$. Studies reveal that the integration of metal catalysts in MOF facilitate adsorption, close-packing, recyclability and siteisolation. These novel structures also permit the chemical and physical functionality conferred by the linkers.

Porphyrin is a purple coloured naturally occurring organic chromophore with flexible photo physical properties. It has been used for various applications in photodynamic therapy for cancer treatments, in dye sensitized solar cells, and can also be applied in creating multifunctional supra molecular structures [7]. It appeals to the scientific community because it allows directed functionalization by attaching pendant molecules and offers selective absorption. A metalated porphyrin MOF lacks non-accessible bulk volume therefore it is a single site catalyst with $100 \%$ utility of active sites and thermally stable microporosity. Accounting from literature, they are structurally similar to cofactors present in many metallo-enzymes. Metallo-porphyrins are thus, potential building blocks for catalytically active MOF.

Amoxicillin belongs to the $\beta$ - Lactam family of Penicillin antibiotic. It is employed in the treatment of various bacterial infections like those of ear, nose, or throat etc. It is preferred due to the ease of administration and wide-spectrum of antibacterial activity. Recent analysis shows that its concentration has escalated to approximately $1.50 \mu \mathrm{g} \mathrm{L}^{-1}{ }^{1}, 2$, Conventional treatments for instance filtration, centrifugation, advanced oxidation processes, activated carbon adsorption and flocculation etc. give rise to secondary contaminants and have very low degradation and removal efficiency. Recently researchers have studied various techniques to degrade antibiotics through ozonation, Fenton and Photo-Fenton processes, although these offer a high degree of degradation but are not feasible in a limited span of time $[8,9]$. Fenton and Photo Fenton processes require nonnegotiable conditions like acidic environment, high ferrous concentrations which are difficult to realize $[10,11]$. Another study involving advanced oxidation process exhibits gradual increase in degradation but is not very promising at ambient temperatures. It requires temperatures above $60^{\circ} \mathrm{C}$ to show proper degradation [12]. Whereas, a recent work employs the use of $\mathrm{ZnO}$ nanoparticles for photo catalysis, but the biggest limitation was that this process could not improve the biodegradability $[13,14]$.

We herein report the solvo thermal synthesis of MOF and its characterization using UV, FTIR, SEM, EDX, XRD etc. We have systematically studied the effect of concentrations of Zn-porphyrin MOF and time duration on degradation of amoxicillin at room temperature under ambient condition in absence of light. The study also presents a comparative overview between three species of MOFs namely, naphthalene based, Manganese based and Zinc porphyrin based MOF, in order to reckon the more efficient one when it comes to drug degradation studies.

\section{Experimental}

\subsection{Materials and Methods}

Zinc acetate (99+\%), Tetra (4-carboxyphenyl) porphyrin H2TCPP (>97\%), Benzoic acid (99\%), dimethylformamide (DMF) $(99+\%)$, were purchased from Sigma Aldrich and used without alterations. Amoxicillin antibiotic was obtained from Mankind pharma. All other chemicals are of analytical grade and from and used without further purification. Deoxidized water used in all experiments was purified by a Mili-Q system.

\subsection{Instrumentation}

The UV-vis spectroscopy was done by Shimadzu uv-vis spectrophotometer UV-1800.. The electrochemical studies were probed using an Autolab Potentiostat / Glavanostat Model AUT83945 (PGSTAT302N). Consequently, cyclic voltammetry was conducted using three electrode cell wherein ITO was the working electrode, Platinum wire acted as counter electrode and $\mathrm{Ag} / \mathrm{AgCl}$ was the reference electrode in PBS (50 mM pH 7.5) containing $5 \mathrm{mM}\left[\mathrm{Fe}(\mathrm{CN})_{6}\right]^{3-/ 4-}$ as a redox species. Autolab Potentiostat/Glavanostat Model AUT83945 (PGSTAT302N) was used for the CV and DPV studies. The FTIR studies were carried out using FTIR from Perkin Elmer Frontier ATR/IR and XRD was performed by Bruker D8 with $\mathrm{Cu} K \alpha$ radiation $(\lambda=0.15406 \mathrm{~nm})$, and the data were collected in the $2 q$ range of $5-30^{\circ}$.

\subsection{Synthesis of Zn-Porphyrin MOF}

The solvo thermal synthesis of $\mathrm{Zn}$ - porphyrin MOF was carried out in absence of light, pertaining to the degradable behavior of porphyrin ligand in presence of visible light. Initially 0.02 moles of $\mathrm{ZnC}_{4} \mathrm{H}_{6} \mathrm{O}_{4}$ were mixed with $50 \mathrm{mg}$ of H2TCPP in a $50 \mathrm{ml}$ Teflon Cell (acetone dried). Furthermore, $3 \mathrm{~g}$ of benzoic acid was added to the above mixture as a modulating agent with consequent addition of $9 \mathrm{ml}$ of DMF. The reaction vial was sonicated for 10 minutes and heated using a Teflon hydrothermal bomb at $120^{\circ} \mathrm{C}$ for 12 hours. The product was collected using ethanol and centrifuged at $8000 \mathrm{rpm}$. The later was washed with acetone and left for air drying while cautiously protecting it from light. This method affords purple coloured microcrystalline powders yielding about $0.118 \mathrm{~g}$ of lab synthesized MOF.

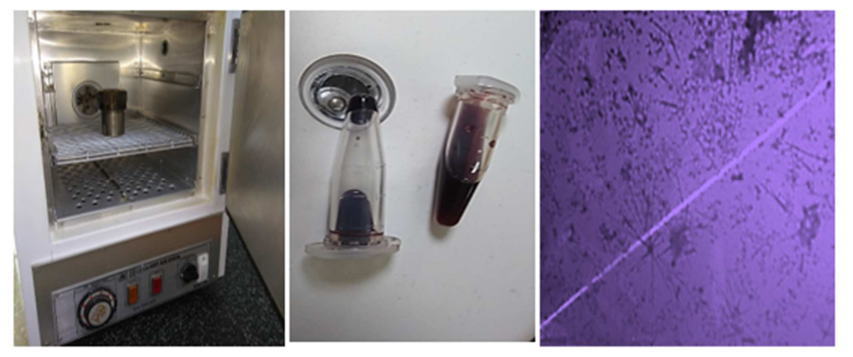

Figure 1. Photographic images of MOF synthesis (a)-Reactants put in hydrothermal bomb, (b)- 0.2 mg precipitate taken for centrifuge@8000 rpm. (c)- Ultra microscopic image of MOF". 


\subsection{Method of Degradation}

Powdered MOF was dissolved in Phosphate buffered saline and $0.1 \mathrm{mg} / \mathrm{ml}$ of antibiotic solution in DI water was used as a stock solution, to analyze the concentration. Various concentrations like 30, 50, $100 \mathrm{ppm}$ of amoxicillin solutions were prepared from stock solution.

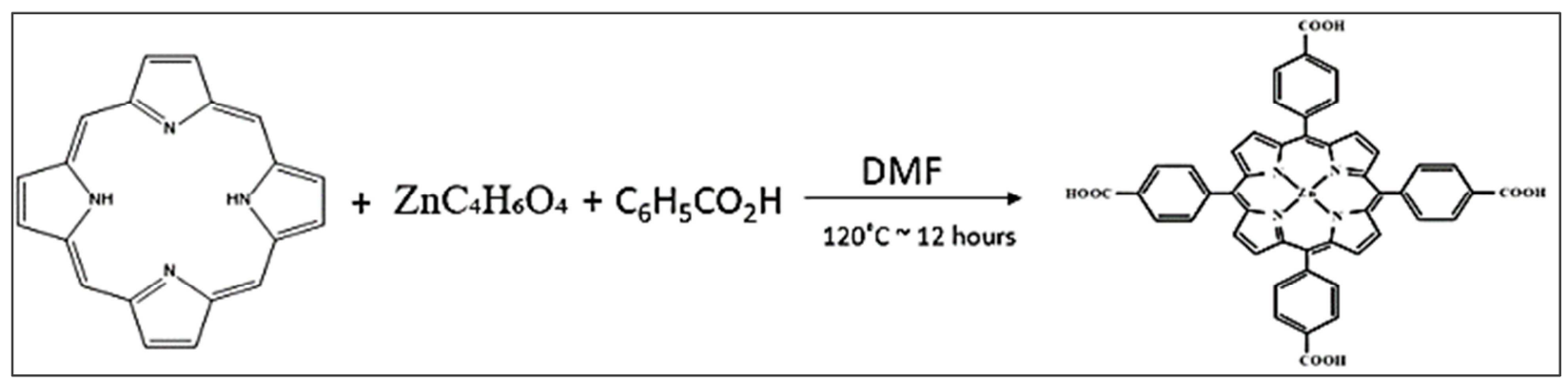

Figure 2. Illustrating solvo thermal synthesis of Zinc-Porphyrin MOF using zinc acetate, benzoic acid and DMF $\sim 120^{\circ} \mathrm{C}$ overnight".

In order to understand the action of MOF on amoxicillin, $0.1 \mathrm{mg}$ of MOF in $1 \mathrm{ml}$ PBS was charged into $1 \mathrm{ml}$ of antibiotic solution in all vials of different concentrations. The mixture was kept under constant stir in a dark environment at room temperature. From the Teflon cell, $200 \mu \mathrm{L}$ of the solution was extracted for further analysis at different time intervals such as 0 mins, 15 mins, 30 mins, 60 mins, 90 mins and 120 mins and degradation efficiency was quantified. Before this, linear calibration curves were developed by plotting the signal intensity of Cyclic Voltammetry, FTIR and DPV from pure amoxicillin at various concentration. Further, signal intensity of residual concentration of Amoxicillin extracted at various time interval from reaction mixture was measured and calculated from the calibration curve.

\section{Results and Discussion}

\subsection{Characterization of Zn-Porphyrin}

The functional elucidation of pure ligand and MOF was carried out by FTIR. Pure Amoxicillin absorption peaks are also noted to analyze the degradation process. For FTIR analysis, pure amoxicillin was scanned over a range of 400 $4500 \mathrm{~cm}^{-1}$ using the FTIR instrument. The band observed at $1635 \mathrm{~cm}^{-1}$ corresponds to $\mathrm{C}=\mathrm{O}$ stretching vibrations. The strong IR absorption is because of the significant change in the atomic dipole moment produced by the stretching vibrations.

UV-vis spectrum for Zn-Porphyrin-MOF reveals strong soret bands at $415 \mathrm{~nm}$ due to $\pi$ to $\pi^{*}$, a sharp peak at $433 \mathrm{~nm}$ with series of Q bands at $521 \mathrm{~nm}, 560 \mathrm{~nm}, 593 \mathrm{~nm}$ and 650 $\mathrm{nm}$ [15]. The shift towards broader wavelength compared to pure ligand indicates creation of bonds that retain the porous structure, this can be validated through Kasha's excitation theory indicating metal aggregation in porphyrin undergoes effective $\pi-\pi$ interaction. Thereby the red shift in the Q bands suggests the formation of the hybrid, this is primarily due to the electron dipole moment that allows $\pi-\pi$ transition in the newly formed MOF [16].

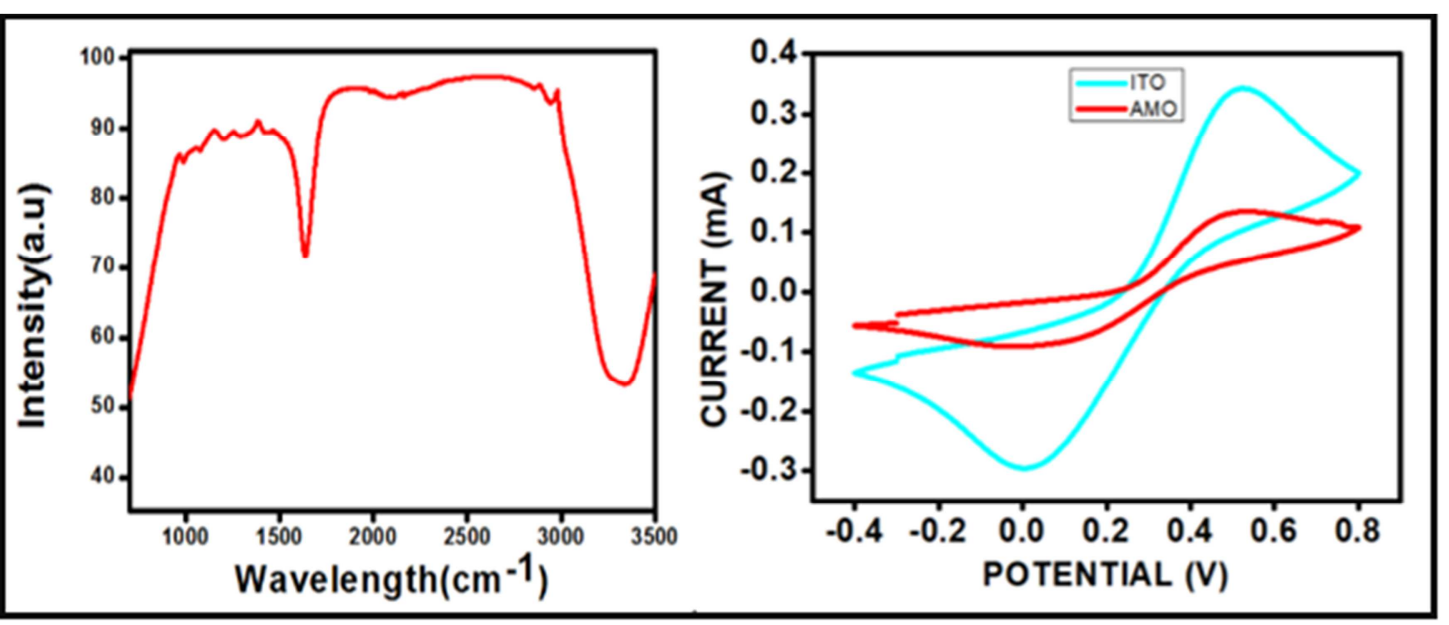

Figure 3. (a): FTIR spectrum of pure amoxicillin antibiotic at room temperature. (b) Cyclic voltammogram of Amoxicillin was analyzed against bare ITO (working electrode), $\left[\mathrm{Fe}(\mathrm{CN})_{6}\right]^{3-14}$ redox probe, Platinum wire (counter electrode) and saturated $\mathrm{Ag} / \mathrm{AgCl}$ (reference electrode) in PBS (50 Mm, pH 7.0, $0.9 \%$ $\mathrm{NaCl}$ ). The scan rate was $100 \mathrm{mV} / \mathrm{s}$. Peak current intensity: Bare ITO $=3.422 \times 10^{-4} \mathrm{~mA}$ and that of amoxicillin is $1.351 \times 10^{-4} \mathrm{~mA}$ ".

The FTIR spectra of the ligand shows characteristic peak depicting $\mathrm{C}-\mathrm{O}$ bond along the broad band at $1200 \mathrm{~cm}^{-1}-1300$ $\mathrm{cm}^{-1}$ [17], furthermore the spectra plunges at $1700 \mathrm{~cm}^{-1}$ indicating $\mathrm{C}=\mathrm{O}$ bond. The $\mathrm{N}-\mathrm{H}$ bond stretching and bending frequency of pure porphyrin ligand can be located at $960 \mathrm{~cm}^{-}$ ${ }^{1}$ which disappears after incorporation of zinc in porphyrin 
ring, and a characteristic $\mathrm{Zn}-\mathrm{N}$ peak is observable instead [18]. Wherein that of the MOF complex exhibits characteristic peaks emerging from the ionic framework.

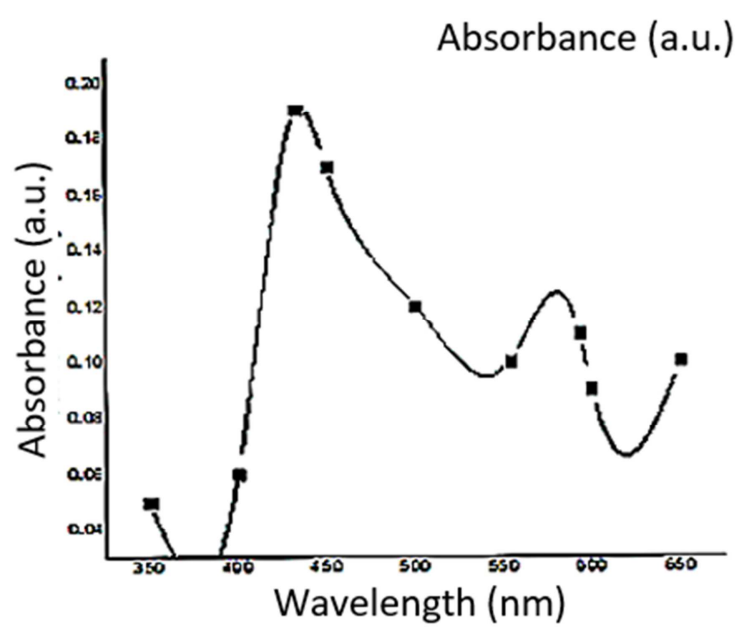

Appearance of the $\mathrm{C}=\mathrm{N}$ stretching peak at $1689 \mathrm{~cm}^{-1}$ indicates the incorporation of $\mathrm{Zn}$ in the $1 \mathrm{D}$ channels of H2TCPP.

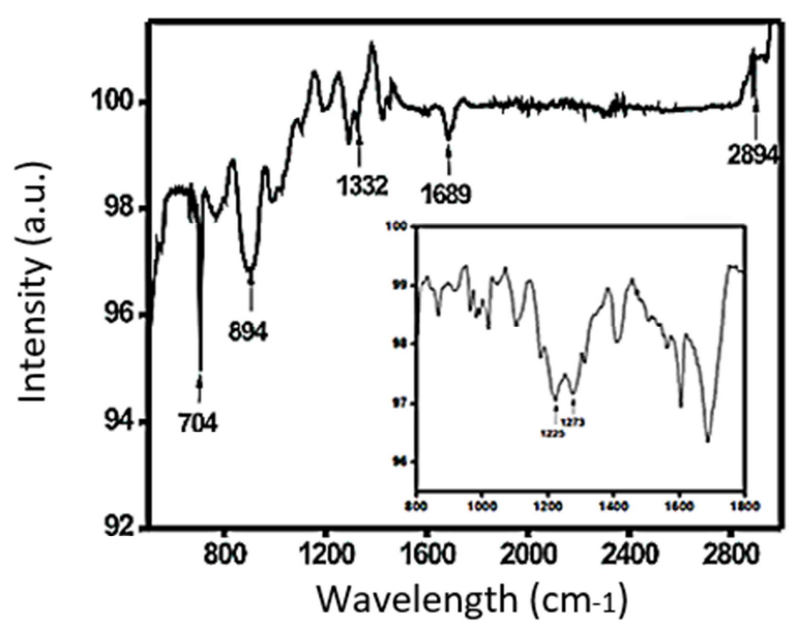

Figure 4. Functional analysis of Zinc porphyrin MOF (a) UV-vis adsorption spectra (b) FTIR spectra of MOF with characteristic peaks showing Zinc and ligand uptake, inset: FTIR of pure porphyrin ligand (recorded at room temperature)".

Characteristics of ligand uptake by the MOF can be studied through the slight redshifts in the plot indicating formation of $\mathrm{Zn}-\mathrm{O}-\mathrm{C}$ linkages at $1100 \mathrm{~cm}^{-1}$ as a result of displacement of carboxyl bonds in porphyrin [19]. The sharp dips in the lower wavelength range are metal dependent and are attributed to vibrations of nitro group. For instance, emergence of $\mathrm{Zn}-\mathrm{N}$ pyrrole ring stretching peak at $704 \mathrm{~cm}^{-1}$ implies the presence of $\mathrm{Zn}-\mathrm{N}$ linkage [20].

\subsection{Imaging Techniques}

The microstructure analysis of Zn Porphyrin MOF was carried using SEM, XRD and EDS techniques. Morphology of $\mathrm{Zn}$ Porphyrin MOF crystals at various time interval during the degradation process were analyzed to comprehend the extent of porosity at 0 mins and then when antibiotic degradation initiates. The architecture as visualized is a robust crystalline MOF structure with porosity more than $50 \%$ of the crystal volume. While comparing the surface morphology of MOF crystal at various time interval of degradation process, Zn-MOF remains unchanged in the degradation process, strongly supporting catalytic nature.
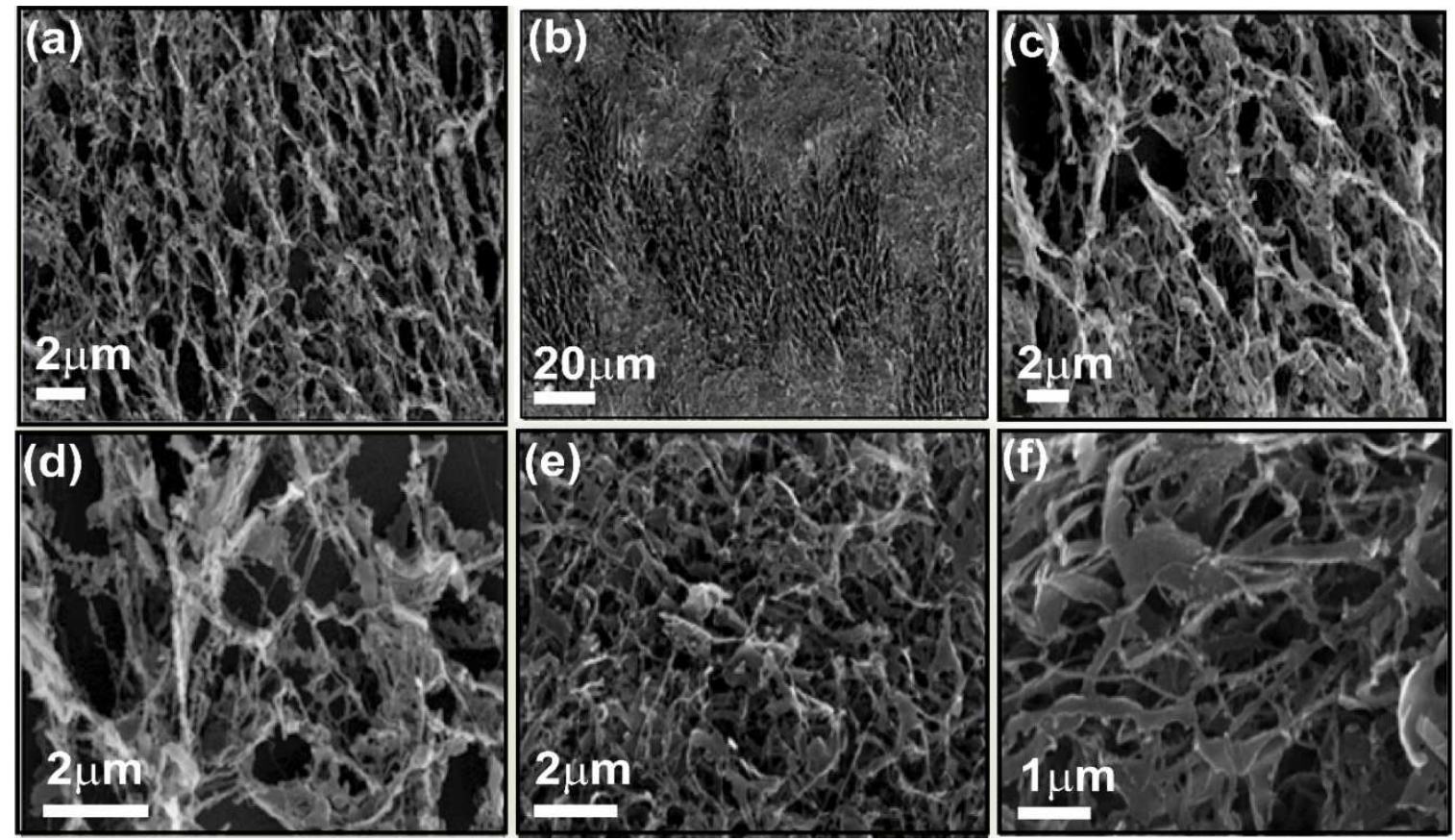

Figure 5. FESEM images showing antibiotic degradation inside the Zn-MOF framework at room temperature. (a) Pristine MOF exhibiting porous sites (0 min.) [magnification $2 \mu \mathrm{m}$ ]. (b) 30 minutes after addition of amoxicillin [mag. $20 \mu \mathrm{m}$ ] (c) 50 min. amx. tends to bind inside the porous sites [mag. $2 \mu \mathrm{m}$ ] (d)

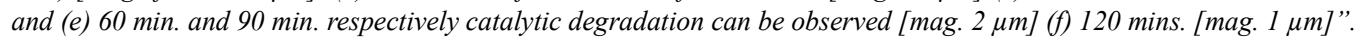




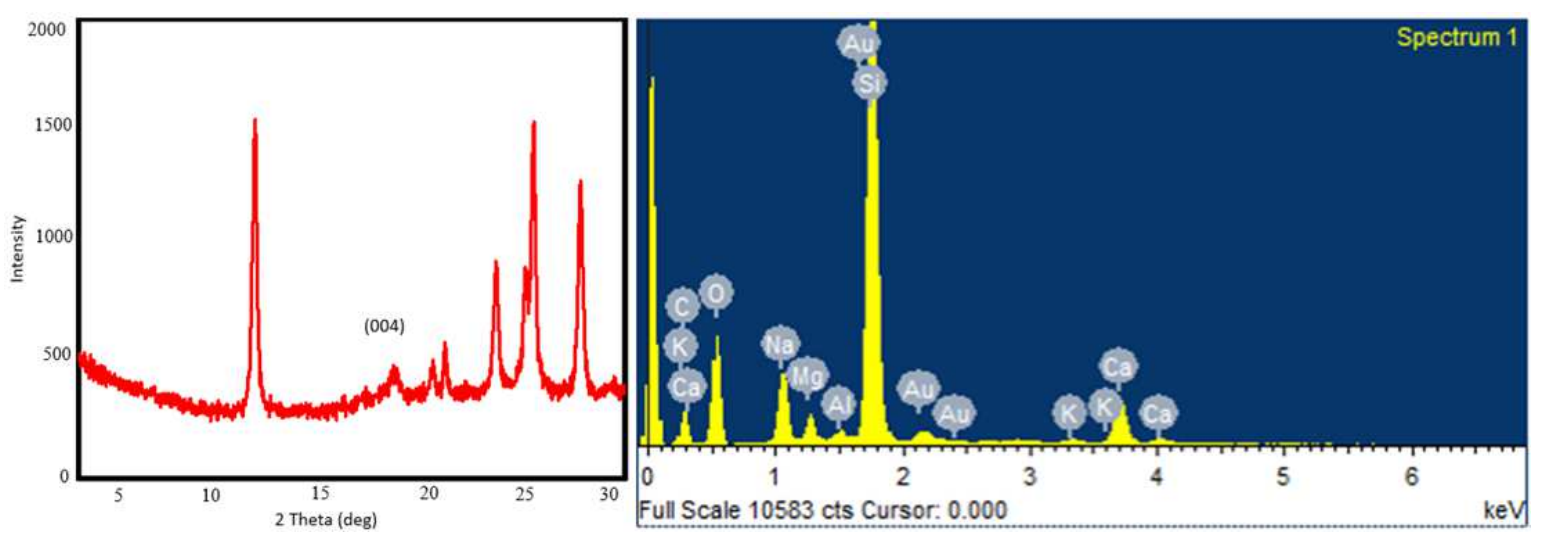

Figure 6. (a) XRD pattern of the as prepared Zn Porphyrin MOF (matched with reported literature) (b) EDS images of the MOF showing metal and ligand components [magnification $30 \mu \mathrm{m}$ ]".

The XRD data reveals characteristic peaks implicating the metalation of porphyrin with zinc ion. A broad diffraction peak at $17.4^{\circ}$ appears for the MOF corresponding to (004) plane [21], while another broad peak at $26^{\circ}$ is indexed as (002) confirming $\mathrm{ZnO}$ linkage [22]. According to Bragg's equation, the calculated interlayer spacing becomes $0.5 \mathrm{~nm}$ which is at par the theoretical value thereby proving conformatory changes in TCPP. For elemental analysis of MOF, Energydispersive X-ray spectroscopy (EDS) was done. The spectrum on processing showed the presence of $\mathrm{CaCO}_{3}, \mathrm{SiO}_{2}, \mathrm{Na}, \mathrm{Mg}$, $\mathrm{Al}_{2} \mathrm{O}_{3}, \mathrm{Si}, \mathrm{K}, \mathrm{Ca}$ and $\mathrm{Au}$. It is done for chemical characterization of the sample as well as to test elemental abundance. Due to very low concentration $\mathrm{Zn}$ is not visible.

\subsection{Degradation Study of Amoxicillin}

Cyclic Voltammetry, FTIR and DPV results were accumulated to determine the course of antibiotic degradation. Cyclic Voltammetry studies were performed using an Autolab Potentiostat/ Galvanostat Model using the three electrode system. There are distinctive reduction and oxidations peaks observed on passing current through the batch samples. From the CV plot between amoxicillin and bare ITO we deduced that amoxicillin is insulating in nature pertaining to the flat curve (no oxidation or reduction peaks), therefore $\mathrm{Fe}[\mathrm{CN}]^{6-3 /-4}$ was taken in electrolyte as a redox probe to pin point the signal intensity. Similarly, when we analyse the MOF-antibiotic solution at 0 minutes there is an immediate drop in current implying that MOF is non conducting as well. On compiling the results for all the batches we can establish that there is an overall increase in the current value recorded over a period of 120 minutes, in 6 batches. After which the current saturates indicating no further degradation or structural alterations. The CV curve shows a minimum peak current intensity of $0.17 \times 10^{-4} \mathrm{~mA}$ whereas the maximum peak current value recorded was 0.54 x $10^{-4} \mathrm{~mA}$ at 0 minutes and 120 minutes respectively. This variation in current suggests that amoxicillin degrades in the presence of $\mathrm{Zn}$ - porphyrin MOF (Figure 7). Moreover, this disintegration assisted conductivity within the structure, which can also be related to structural changes in amoxicillin. These results were later verified by FTIR and DPV findings.
The FTIR spectrum of Amoxicillin demonstrates a characteristic peak at $1635 \mathrm{~cm}^{-1}$ which is observed in the degradation study, along with other peaks. The trend in transmittance percentage takes a steady rise from 0 to 90 minutes implying that amoxicillin degrades with increased exposure to MOF and it drops suddenly at 120 minutes indicating that no further degradation is observed. While the other peaks follow an analogous course, the spectra at 30 minutes arrays a marginal decline in absorbance when compared to 0 minutes. At 60 minutes we recorded two unique peaks $1427 \mathrm{~cm}^{-1}$ and $1290 \mathrm{~cm}^{-1}$ assigned for $\mathrm{C}-\mathrm{N}$ and $\mathrm{C}-\mathrm{O}$ bond respectively.

The maximum transmittance for the characteristic peak is reported at 90 minutes which simply indicates maximal degradation. While the peaks corresponding to amoxicillin composition demonstrate variation in transmittance, those characteristic for MOF retain their transmittance values. For instance, $704 \mathrm{~cm}^{-1}, 1689 \mathrm{~cm}^{-1}, 1332 \mathrm{~cm}^{-1}$ which specify Zn$\mathrm{N}, \quad \mathrm{C}-\mathrm{C}$ and $\mathrm{C}-\mathrm{N}$ bonds respectively show sustained transmittance values even at 120 minutes (Figure 8). Thereby the observations of $\mathrm{CV}$ and FTIR tests are relatable as increase in transmittance corresponds to degradation of antibiotic with increase in peak current.

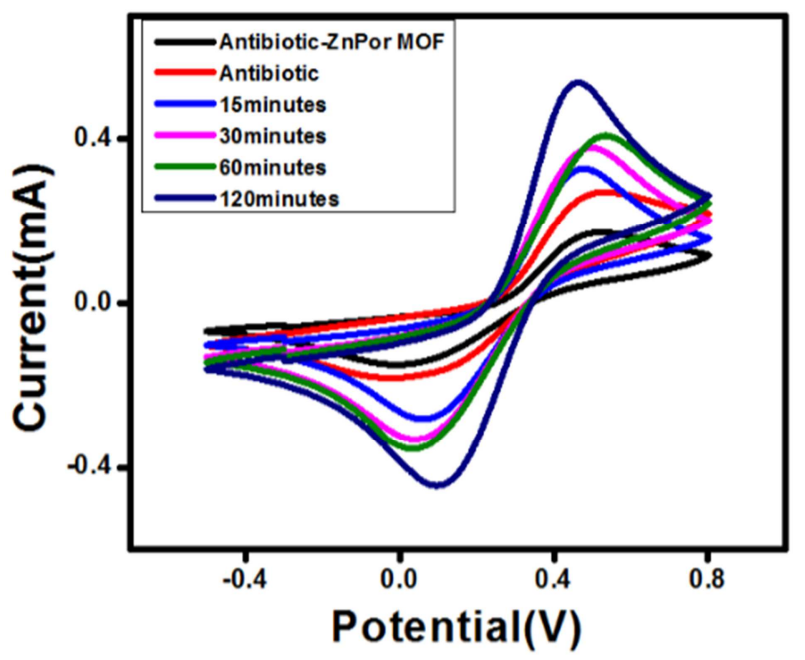

Figure 7. CV Analysing the degradation of amoxicillin where working electrode is bare ITO, platinum (Pt) wire is auxiliary electrode and saturated $\mathrm{Ag} / \mathrm{AgCl}$-reference electrode, in PBS (50 $\mathrm{Mm}, \mathrm{pH} 7.0,0.9 \% \mathrm{NaCl})$ containing $5 \mathrm{mM}\left[\mathrm{Fe}(\mathrm{CN})_{6}\right]^{3-/ 4-}$ as redox mediator". 


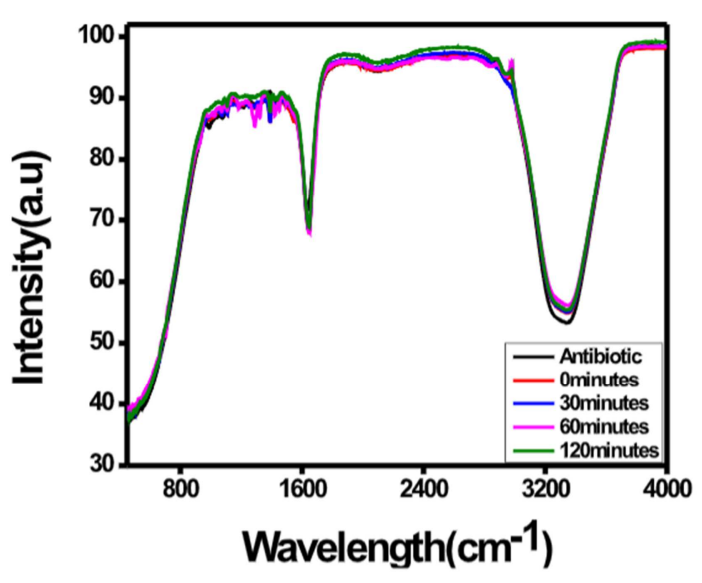

Figure 8. FTIR spectra representing the degradation of amoxicillin over a period of 120 minutes. It was studied in the range of $800-1700 \mathrm{~cm}^{-1}$. All tests were carried in absence of light at room temperature and the characteristic amx. peak $1635 \mathrm{~cm}^{-1}$ was followed for degradation analysis".

In order to estimate the total time for degradation DPV studies were conducted. DPV is very sensitive, such that it confers direct analyses at ppb (parts per billion) level. Moreover, in a stripping mode, parts per trillion analyse are also feasible. Detectability is due to the relatively short pulse time and its differential nature. The short pulse time increases the measured currents, while the differential analysis differentiates amongst background processes. The sharp reversal of peak depicts degradation and valley measures the time lapsed for maximal breakdown.

The Kinetics study for the reaction gave a straight line graph as that of first order reactions, it corresponds to the trend of change in concentration with respect to time. The slope of the graph was further employed to estimate the Eq. constant value for the reaction, as a result of which we found that the Eq. constant $=0.0381944$ [Equation Eq. Constant $=$ slope*2.303].

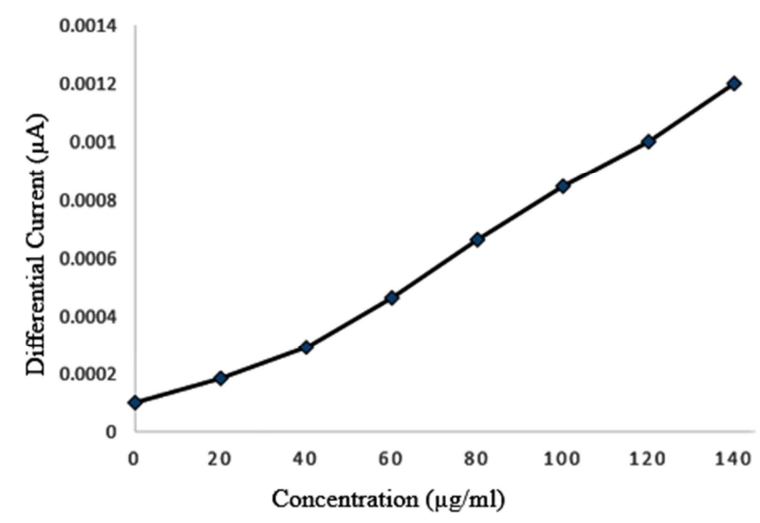

Figure 9. Differential pulse voltammogram recorded for degradation of amoxicillin with the Zn-porphyrin system at room temperature".
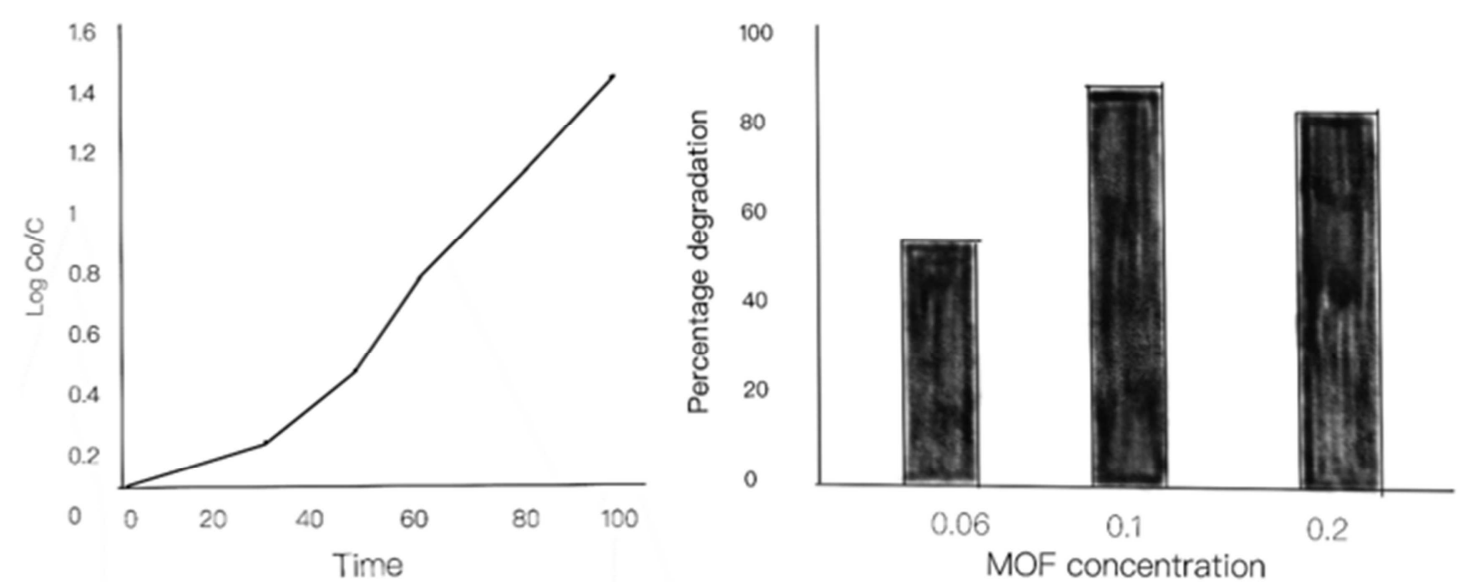

Figure 10. (a) Kinetics study of the process shows that the degradation follows a first order model [time: in min] (b) Study about the effect of catalyst loading for constant amount of amoxicillin"

\subsection{Catalytic Behavior OF Zn Porphyrin MOF}

The estimation of degradation efficiency of MOF was carried by analysing the effect of different MOF concentration on $50 \mathrm{ppm}$ antibiotic concentration. Furthermore, we employed the mathematical formula to justify this result wherein, degradaion efficiency was equivalent to; Catalytic Eff. $=\left[\mathrm{C}_{\mathrm{t}}-\mathrm{C}_{0} / \mathrm{C}_{0} * 100\right]$

\{Where $\mathrm{C}_{\mathrm{t}}$ : Concentration at a given time, $\mathrm{C}_{0}$ : Initial Concentration $\}$

Thus the degradation effiicency is estimated to be $97.337 \%$.

Simultaneously titrations with $\mathrm{KMnO}_{4}$ were carried out to estimate the catalytic potential. The investigation reported that for degradation of approximately $80 \mathrm{ppm}$ amoxicillin $0.1 \mathrm{mg}$
MOF is required, the reaction occurs in absence of light and has and regardless of the original concentration, complete antibiotic degradation can be achieved within 90 minutes. Additionally, the stability of the catalyst after the antibiotic has been degraded can be precisely spotted by the presence of characteristic $\mathrm{CN}$ peak at $1427 \mathrm{~cm}^{-1}$. This matches with the functional peaks of the parent MOF thus supporting the assumption that the MOF will retain its original morphology.

\subsection{Comparative Study Between Various MOFs}

In view of investigating the amount of antibiotic that degenerates after the maximum time of degradation is achieved, we opted for titration methodology. Apart from that three species of MOFs were evaluated and later classified on 
the basis of degradation potential. Although Naphthalene based MOF was ruled out of the investigation pertaining to the fact that it is susceptible to structural collapse upon removal of matrix phase. Moreover, not only the synthesis was a marathon of procedures but the UV-vis spectra do not demonstrate a sharp characteristic peak for Naphthalene ligand [23]. The disappearance of Antimicrobial drug per unit of porphyrin was evaluated by conducting titration experiments with $\mathrm{KMnO}_{4}$. The titration of $100 \mathrm{ppm}$ antibiotic solution, where the reported volume of amoxicillin was $20 \mathrm{ml}$ in $0.01 \mathrm{M}$ of $\mathrm{KMnO}_{4}$ solution reveals that $0.00015 \mathrm{M}$ of Amoxicillin was found intact in the case of Manganese based MOF, whereas only $0.000075 \mathrm{M}$ was extracted unaltered in case of $\mathrm{Zn}$-Porphyrin based MOF. Therefore, it can be ascertained that approximately $54 \mathrm{ppm}$ of antibiotic was left unaltered when exposed to degradation by $\mathrm{MnCl}_{2}$ - MOF. Whereas barely 27.4 ppm of pristine antibiotic composition was reported after the degradation activity by $\mathrm{Zn}$ Porphyrin-MOF.

\section{Conclusion}

The paper unfolds a pristine area reporting the application of MOF for antibiotic degradation. Herein, we fabricated zinc Porphyrin MOF and thoroughly characterized it by employing UV-Vis spectroscopy, SEM, EDS, XRD, CV and FTIR. The MOF thus curated is a stable structure with $\pi-\pi$ interactions, sustaining high porosity values, has completely accessible metalloporphyrin sites and shows catalytic activity in the interior of the material. Furthermore, we employed the catalyst to measure its ability of amoxicillin degradation in absence of light. The results from FTIR and CV studies are relatable and reveal steady degradation of active antibiotic between 30 to 90 minutes of exposure. The kinetics study revealed that the degradation process follows a 1st order dependence on antibiotic concentration. The peak at 1290 $\mathrm{cm}^{-1}$ (120 minutes) implies conformational changes in antibiotic with transformation into $\mathrm{C}-\mathrm{O}$ bond. The degradation efficiency of MOF was evaluated by studying the effect of change in catalyst loading in $100 \mathrm{ppm}$ amoxicillin solution, demonstrating about $97.33 \%$ of catalytic efficiency. We have also accounted that $0.1 \mathrm{mg}$ of Zinc porphyrin MOF is capable of degrading $100 \mathrm{ppm}$ amoxicillin antibiotic within 90 minutes.

Since the original morphology of the MOF remains intact and the deduction that even after complete degradation antibiotic does not configure into a non-biodegradable product, this approach can be a pioneer towards realizing a MOF with rapid catalytic activity. Remarkably, the porous nanocomposite acts as an efficient bio-friendly heterogeneous catalyst for degradation of the sequestered amoxicillin.

\section{References}

[1] Guo, W.; Su, S.; Yi, C.; Ma, Z. Degradation of Antibiotics Amoxicillin by Co 3 O 4 -Catalyzed Peroxymonosulfate System. Environ. Prog. Sustain. Energy 2013, 32 (2), 193197. https://doi.org/10.1002/ep.10633.
[2] Dimitrakopoulou, D.; Rethemiotaki, I.; Frontistis, Z.; Xekoukoulotakis, N. P.; Venieri, D.; Mantzavinos, D. Degradation, Mineralization and Antibiotic Inactivation of Amoxicillin by UV-A/TiO 2 Photocatalysis. J. Environ. Manage. 2012, $98 \quad$ (1), 168-174. https://doi.org/10.1016/j.jenvman.2012.01.010.

[3] Roy, R.; Tropper, F. D.; Romanowska, A. New Strategy in Glycopolymer Syntheses. Preparation of Antigenic WaterSoluble Poly (Acrylamide-Co-p-Acrylamidophenyl. Beta.Lactoside). Bioconjug. Chem. 1992, 3 (3), 256-261. https://doi.org/10.1021/bc00015a009.

[4] M. Visalakshi, G. Susheela Bai, V. Sinduri, G. Praveena, G. Ushasree and G. Swapna, "Photocatalytic degradation of outdated antibiotics by nano-crystalline $\mathrm{ZnO}$," International Conference on Advanced Nanomaterials \& Emerging Engineering Technologies, Chennai, 2013, pp. 409-411, doi: 10.1109/ICANMEET.2013.6609331.

[5] Wade, C. R.; Li, M.; Dincă, M. Facile Deposition of Multicolored Electrochromic Metal-Organic Framework Thin Films. Angew. Chemie 2013, 125 (50), 13619-13623. https://doi.org/10.1002/ange.201306162.

[6] Senkovska, I.; Hoffmann, F.; Fröba, M.; Getzschmann, J.; Böhlmann, W.; Kaskel, S. New Highly Porous Aluminium Based Metal-Organic Frameworks: $\mathrm{Al}(\mathrm{OH})(\mathrm{Ndc})(\mathrm{Ndc}=2,6$ Naphthalene Dicarboxylate) and $\mathrm{Al}(\mathrm{OH})(\mathrm{Bpdc})\left(\mathrm{Bpdc}=4,4^{\prime}-\right.$ Biphenyl Dicarboxylate). Microporous Mesoporous Mater. 2009, 122 (1-3), 93-98. https://doi.org/10.1016/j.micromeso.2009.02.020.

[7] Son, H. J.; Jin, S.; Patwardhan, S.; Wezenberg, S. J.; Jeong, N. C.; So, M.; Wilmer, C. E.; Sarjeant, A. A.; Schatz, G. C.; Snurr, R. Q.; Farha, O. K.; Wiederrecht, G. P.; Hupp, J. T. Light-Harvesting and Ultrafast Energy Migration in Porphyrin-Based Metal-Organic Frameworks. J. Am. Chem. $\begin{array}{llll}\text { Soc. } & 2013, & 135 & \text { (2), }\end{array}$ https://doi.org/10.1021/ja310596a.

[8] Ay, F.; Kargi, F. Advanced Oxidation of Amoxicillin by Fenton's Reagent Treatment. J. Hazard. Mater. 2010, 179 (13), 622-627. https://doi.org/10.1016/j.jhazmat.2010.03.048.

[9] Elmolla, E. S.; Chaudhuri, M. Degradation of the Antibiotics Amoxicillin, Ampicillin and Cloxacillin in Aqueous Solution by the Photo-Fenton Process. J. Hazard. Mater. 2009, 172 (23), 1476-1481. https://doi.org/10.1016/j.jhazmat.2009.08.015.

[10] Sun, J.; Li, X.; Feng, J.; Tian, X. Oxone/Co2+ Oxidation as an Advanced Oxidation Process: Comparison with Traditional Fenton Oxidation for Treatment of Landfill Leachate. Water Res. 2009, 43 (17), 4363-4369. https://doi.org/10.1016/j.watres.2009.06.043.

[11] Andreozzi, R.; Canterino, M.; Marotta, R.; Paxeus, N. Antibiotic Removal from Wastewaters: The Ozonation of Amoxicillin. J. Hazard. Mater. 2005, 122 (3), 243-250. https://doi.org/10.1016/j.jhazmat.2005.03.004.

[12] Xie, M. H.; Yang, X. L.; Zou, C.; Wu, C. De. A SnIVPorphyrin-Based Metal-Organic Framework for the Selective Photo-Oxygenation of Phenol and Sulfides. Inorg. Chem. 2011, 50 (12), 5318-5320. https://doi.org/10.1021/ic200295h.

[13] Iranmanesh, P.; Saeednia, S.; Nourzpoor, M. Characterization of $\mathrm{ZnS}$ Nanoparticles Synthesized by Co-Precipitation Method. Chinese Phys. B 2015, 24 (4), 1-4. https://doi.org/10.1088/1674-1056/24/4/046104. 
[14] Gong, Y.; Wu, T.; Lin, J. Metal-Organic Frameworks Based on Naphthalene-1, 5-Diyldioxy-Di-Acetate: Structures, Topologies, Photoluminescence and Photocatalytic Properties. CrystEngComm 2012, 14 (10), 3727-3736. https://doi.org/10.1039/c2ce06529k.

[15] Gao, Y.; Zhang, X.; Ma, C.; Li, X.; Jiang, J. MorphologyControlled Self-Assembled Nanostructures of 5, 15-Di [4-(5Acetylsulfanylpentyloxy) Phenyl] Porphyrin Derivatives. Effect of Metal-Ligand Coordination Bonding on Tuning the Intermolecular Interaction. J. Am. Chem. Soc. 2008, 130 (50), 17044-17052. https://doi.org/10.1021/ja8067337.

[16] Zhao, Y.; Cai, X.; Zhang, Y.; Chen, C.; Wang, J.; Pei, R. Porphyrin-Based Metal-Organic Frameworks: Protonation Induced Q Band Absorption. Nanoscale 2019, 11 (25), 12250 12258. https://doi.org/10.1039/C9NR02463H.

[17] Sun, Z. C.; She, Y. Bin; Zhou, Y.; Song, X. F.; Li, K. Synthesis, Characterization and Spectral Properties of Substituted Tetraphenylporphyrin Iron Chloride Complexes. Molecules 2011, 16 (4), 2960-2970. https://doi.org/10.3390/molecules16042960.

[18] Sharma, N.; Dhankhar, S. S.; Nagaraja, C. M. EnvironmentFriendly, Co-Catalyst- and Solvent-Free Fixation of CO 2 Using an Ionic Zinc (II) -Porphyrin Complex Immobilized in Porous Metal-Organic Frameworks. Sustain. Energy Fuels 2019, 3 (11), 2977-2982. https://doi.org/10.1039/C9SE00282K.

[19] Zhao, W.; Wang, Y.; Wang, A.; Wu, L.; Wang, Q. Facile Synthesis and Photocatalytic Activity of a Novel Titanium
Dioxide Nanocomposite Coupled with Zinc Porphyrin. Nanomater. Nanotechnol. 2016, 6, 184798041666948. https://doi.org/10.1177/1847980416669487.

[20] Sokolov, M. R.; Enakieva, Y. Y.; Yapryntsev, A. D.; Shiryaev, A. A.; Zvyagina, A. I.; Kalinina, M. A. Intercalation of PorphyrinBased SURMOF in Layered Eu (III) Hydroxide: An Approach Toward Symbimetic Hybrid Materials. Adv. Funct. Mater. 2020, 2000681. https://doi.org/10.1002/adfm.202000681.

[21] Zhao, Y.; Jiang, L.; Shangguan, L.; Mi, L.; Liu, A.; Liu, S. Synthesis of Porphyrin-Based Two-Dimensional MetalOrganic Framework Nanodisk with Small Size and Few Layers. J. Mater. Chem. A 2018, 6 (6), 2828-2833. https://doi.org/10.1039/C7TA07911G.

[22] Wang, S.; Shang, L.; Li, L.; Yu, Y.; Chi, C.; Wang, K.; Zhang, J.; Shi, R.; Shen, H.; Waterhouse, G. I. N.; Liu, S.; Tian, J.; Zhang, T.; Liu, H. Metal-Organic-Framework-Derived Mesoporous Carbon Nanospheres Containing Porphyrin-Like Metal Centers for Conformal Phototherapy. Adv. Mater. 2016, 28 (38), 8379-8387. https://doi.org/10.1002/adma.201602197.

[23] Jiang, C. H.; Song, L. F.; Jiao, C. L.; Zhang, J.; Sun, L. X.; Xu, F.; Zhang, H. Z.; Xu, Q. Y.; Gabelica, Z. Determination of Heat Capacities and Thermodynamic Properties of Two Structurally Unrelated but Isotypic Calcium and Manganese (II) 2, 6-Naphthalene Dicarboxylate-Based MOFs. J. Therm. Anal. Calorim. 2011, 103 (3), 1095-1103. https://doi.org/10.1007/s10973-010-1197-7. 